\title{
Effects of Exercise Duration and Number of Players in Heart Rate Responses and Technical Skills During Futsal Small-sided Games
}

\author{
Ricardo Duarte ${ }^{1, *}$, Nuno Batalha ${ }^{1}$, Hugo Folgado ${ }^{1}$ and Jaime Sampaio ${ }^{2}$ \\ ${ }^{1}$ Department of Sport and Health, University of Évora, Portugal \\ ${ }^{2}$ Research Center in Sports Sciences, Health and Human Development (CIDESD), University of Trás-os-Montes e Alto \\ Douro, Portugal
}

\begin{abstract}
In the design of training programs, it is common for coaches to modify task constraints to conduct the drill intensity at the targeted level. Sport specific drills with variations are constantly used in Futsal practices, however the knowledge about how these variations influence drill intensity and technical demands is very scarce. The purpose of this study was to measure the physiological and technical effects of both duration and variations in the numbers of players in futsal specific drills. Heart rates and technical skills of 8 semi-professional futsal players were recorded during four specific drills. The experimental protocol consisted in a half-court game with official rules, played in $4 \mathrm{v} 4,3 \mathrm{v} 3$ and $2 \mathrm{v} 2$, each during 4 minutes and $4 \mathrm{v} 4$ during 10 minutes. It was maintained an active break of 4 minutes between each repetition. The lowest \%HRmax was observed in response to the $4 \mathrm{v} 4$ drill independent of the exercise duration. In players' number variation statistically significant differences were found in the percentage of time spent between $65-85 \% \mathrm{HRmax}$, in the number of successive contacts with the ball and number of dribbles. In exercise duration, statistically significant differences were found in percentage of time spent above $85 \% \mathrm{HRmax}$, in the number of successive contacts with the ball, number of dribbles and number of tackles. The decrease in the number of players and exercise duration resulted in intensity increases and more frequent individual tactical actions.
\end{abstract}

Key Words: Training intensity, Technical demands, Small-sided games, Futsal.

\section{INTRODUCTION}

Futsal is a recent ball team sport with a significant increase in popularity over last years. Because of his genesis, some rules are similar to other sports like soccer, basketball, handball and hockey. It is played on a 40x20m indoor court by 2 teams, each with 5 players and with goals measuring $3 \times 2 \mathrm{~m}$, during two 20 -minute periods where the clock is stopped for some events. The tactical, technical and energetic dimensions of futsal showed evolutionary tendencies that have solidified an own identity through a more thorough specialization $[1,2]$, diverging now from soccer. This idea has been supported time motion analysis research, particularly by identifying mean values of total distance covered and distance covered at different intensities more similar to handball and basketball than to soccer [2].

Available research has demonstrated that Futsal is a multiple-sprint sport in which high-intensity exercise constitutes a greater proportion of match time than in all other team sports [1]. The results indicate a greater proportion of time spent above $85 \%$ of maximum heart rate (HRmax) with mean values of $90 \%$ HRmax per match. Under more specific analysis, only $0.3 \%$ of playing time is spent in low activity (below $65 \%$ of HRmax), $16 \%$ in moderate activity (between $65-85 \%$ of HRmax), and $83 \%$ in very vigorous activity (above $85 \%$ of HRmax) [1]. These cardiovascular demands

*Address correspondence to this author at the Pavilhão Gimnodesportivo da Universidade de Évora, Rua de Reguengos de Monsaraz, 14, 7000-727 Évora, Portugal; Tel: 00351961833206; Fax: 00351266769521;

E-mail: rduarte@uevora.pt demonstrate the primordial role of the anaerobic metabolism which in most players is above the lactate threshold [1].

The intermittent profile of the matches turns high the physical, technical and tactical demands. Despite this information, research concerning training intensity is scarce and remains unclear. One study has investigated the intensity of Futsal specific drills [3], however the studied sample consisted of high school students that played recreational five-aside indoor soccer. The drill consisted of a $5 \mathrm{v} 5$ (including goalkeepers) with $30 \times 15 \mathrm{~m}$ during $30 \mathrm{~min}$. The mean intensity of the drill was $83.5 \pm 5.4 \%$ of $\mathrm{HR}_{\text {peak }}$ with a correspondent $75.3 \pm 11.2 \%$ of $\mathrm{VO}_{2 \text { peak }}$.

In the design of training programs, it is common for coaches to alter some task constraints to conduct the drill intensity at the targeted level $[4,5]$. One preliminary study was conduct to test the influence of pitch size, number of players and consecutive contacts with the ball in relation to the intensity of Futsal specific drills [6]. The results showed a significant increase in drill intensity associated to the increase in court size and reduction in the number of players, probably due to the larger ratio of game space per player, accordingly to the study conducted by Rampinini and colleagues [4] in twelve types of small-sided soccer games. However, another study, centered on soccer drills, challenged this conclusion by showing significant intensity increase associated with smaller court sizes [5]. Another drill variation studied by these authors [5] was centered on drill duration with no significant differences being recorded between 3 and 8 min of practice.

The use of small-sided and conditioning games are also suggested by many authors as an efficient strategy to in- 
crease players specific practice time, eliciting simultaneously physical and technical aspects within a major tactical involvement [4, 5, 7-12]. Notwithstanding the aforementioned, the research methods appear to facilitate the study of the physiologic impact of these drills while proving detrimental to the study of the technical variables. No study has investigated the effects of drill variations in the emergence of technical skills in Futsal specific drills.

Thus, the purpose of this study was to measure the physiological and technical effects of both duration and variations in the numbers of players in Futsal small-sided games. To that end, the percentage of maximal Heart Rate (\%HRmax) and the percentage of time spent in different Heart Rate intensity zones were evaluated while technical skills were measured by notational analysis. It was hypothesized that variations in Futsal specific drills can induce physiological and technical changes in player performance.

\section{METHODS}

\section{Subjects}

The studied sample consisted of eight semi-professional players from a Portuguese First Division team (who volunteered for the study), with a weekly training volume of 630 minutes plus one competition. Mean (SD) age, weight, height, and body mass index were 25.9 (4.5) years, 67.6 (7.5) $\mathrm{kg}, 170(0.1) \mathrm{cm}$, and 22.4 (2.0) percent. All participants, coaches and managers were informed about the procedures and gave their written consent. The study was approved by the Ethical Commission of Investigation in Human Health and Wellness at University of Évora. All participants were familiar with all procedures used prior to the study.

\section{PROCEDURES}

In the week before the gathering process all subjects were measured for weight and height. Participants were asked to maintain their normal diet, which emphasized high fluid and carbohydrate intakes. The data gathering process occurred 75 hours after a weekly competition, in the normal course of the competitive period and in the normally scheduled training time. Participants took part in the training drills as part of their normal training session. Before the start, short-range telemetric heart rate monitors (Polar Team System, Polar Electro, Kempele, Finland) were accurately set in the xiphoid appendix of the players and adjusted, using elastic bands. To maintain normal training, context and experimental conditions, participants were informed of the drill aims and structure by their coaches. They were present and provided encouragement during all drill activities, as was usual in common practice. The session began with the teams' usual warm-up, consisting of patterned collective movements along the entire court without opposition during 10 minutes, followed by 5 minutes of static and dynamic stretch. The studied drill is one of the most common drills practiced by the team, and it consists of the half-court game $(20 \times 20 \mathrm{~m})$ following the official rules, except when the ball went outside the back lines, in which case the goalkeepers restarted the game. Every condition of the exercise lasted for 4 minutes, followed with an active break of 4 minutes during which the players carried out continuous running with low intensity. A flow chart of every drill condition and experimental design is shown in Fig. (1). To ensure that there were minimal stoppages in play during all drill conditions, 4 futsal balls were maintained in each goal to encourage quick restarts in play by the goalkeepers.

Heart rates were monitored telemetrically using 5-second intervals during the training session. After the confirmation of mean differences between active breaks and exercise bouts $(\mathrm{P}<0.05)$, rest periods were excluded from the analysis. Maximal heart rates for each player were estimated through 220 -age, because it minimized underestimating the real values for this specific population [13]. A $25 \mathrm{~Hz}$ video camera was set-up in upper plane to record all drill phases. The video-film was later replayed to evaluate the following tech-

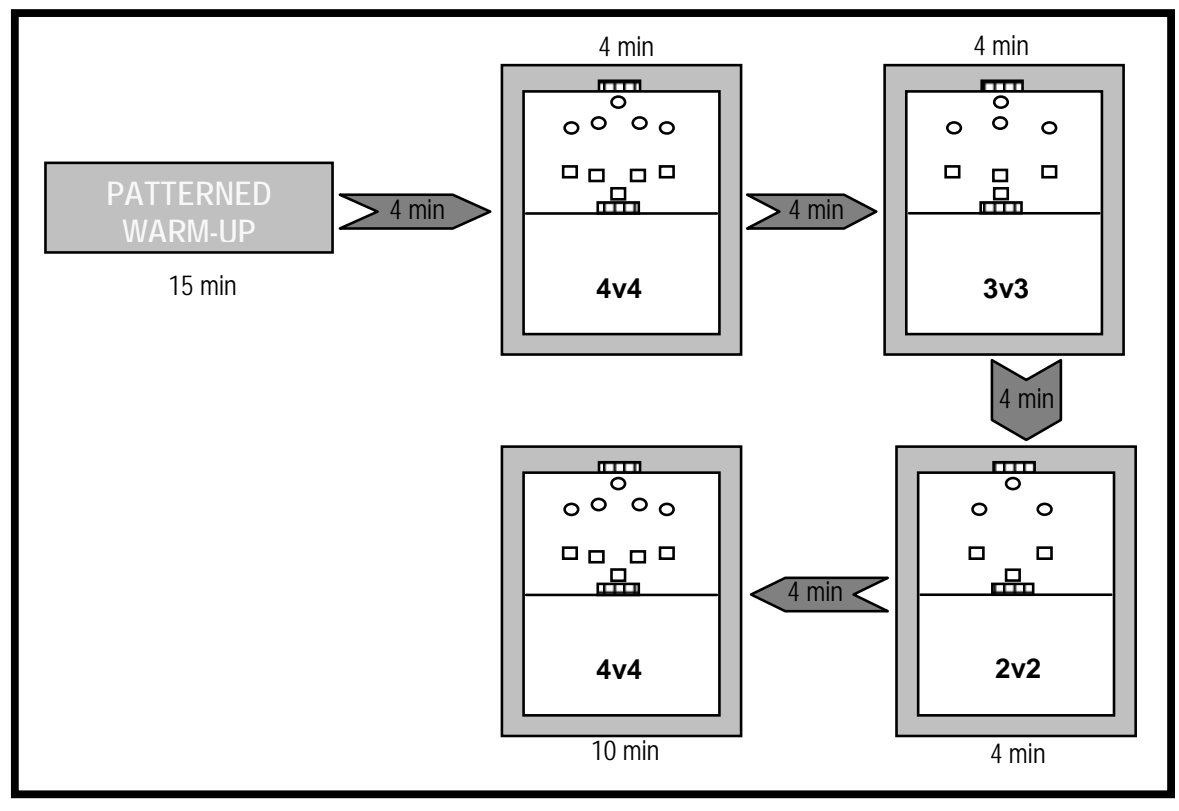

Fig. (1). Experimental protocol. 
nical skills: 1) number of ball possessions; 2) number of successive contacts with the ball; 3) number of dribbles (individual solution); 4) number of correct and wrong passes; 5) number of shots on goal; 6) number of tackles and interceptions. To avoid disagreement among competent observers, the technical skills were scored by a single expert observer who showed $100 \%$ limits of agreement in test-retest after 72 hours for all variables in all drill conditions. Mean percentage of maximum heart rate (\%HRmax), the percentage of time spent in three Heart Rate intensity zones (HRzone1 < 65\%HRmax; HRzone2 = 65-85\%HRmax; HRzone3 > 85\%HRmax) and the mean of technical skills was calculated for each player during all drill conditions.

\section{STATISTICAL ANALYSES}

Repeated measures Anova and non-parametric KruskalWallis Anova were used. When appropriate, Scheffé's post-hoc multiple comparison test was employed. To analyze the time spent in different heart rate intensity zones a Chi-Square test was used. Significance level was maintained at $95 \%(\mathrm{P} \leq 0.05)$.

\section{RESULTS}

The mean $\pm \mathrm{SD}$ responses of $\% \mathrm{HRmax}$ to all drills are shown in Table 1. The lowest \%HRmax was observed in response to the $4 \mathrm{v} 4$ drill independent of the exercise duration, with a significant increase to the $3 \mathrm{v} 3$ and $2 \mathrm{v} 2$.

The time spent in heart rate intensity zones was discriminated and revealed more differences between the studied drill conditions. Fig. (2a) shows statistical differences in time spent in HRzone3 to exercise duration with a significantly superior percentage in the 4-minute condition. Fig. (2b) shows statistical differences in time spent in HRzone2 to players' number variation with more elevated percentage in $4 \mathrm{v} 4$ compared to $3 \mathrm{v} 3$ and $2 \mathrm{v} 2$.

The mean \pm SD of technical data are displayed in Table $\mathbf{1}$. Statistical differences were found in a number of successive contacts with the ball and number of dribbles varying the number of players and exercise duration. Also the number of tackles was statistically different to exercise duration with superior mean values in the 4-minute condition.

\section{DISCUSSION}

It is very common in Futsal practices the use of smallsided games to provide simultaneous skill and fitness training. Sometimes, the coaches have the need to improve the intensity level and/or the density of any technical skill during the training session. Drill variation is a common strategy used by coaches to conduct players to physiological and technical targets. Nevertheless, the scientific basis as to how these variations influence player performance is unknown.

The results of this study suggest that by modifying the number of players and the exercise duration of Futsal specific drills, coaches can change the physiological and technical stimulation of the players. The decrease in the number of players resulted in an intensity increase, probably due to the larger ratio of area per player. This finding is in accord of two studies developed in other team sports [4, 14], and confirms the results of our exploratory study in futsal [6] relative to \%HRmax. Additionally, the analysis of time spent in HRzone 2 reveals that $4 \mathrm{v} 4$ induced a higher percentage of time spent in moderate activity than $3 \mathrm{v} 3$ and $2 \mathrm{v} 2$. Thus, coaches can increase the physiological demands of the drills by reducing the number of players involved. Some technical skills also changed with the variation of the number of players, with significant increases in the number of successive contacts with the ball and the number of dribbles in the $2 \mathrm{v} 2$ drill condition, accordingly to the previous findings of Owen et al. [15] in small-sided soccer games. It is possible that the lower number of choices available for the player with the ball, as he can only cooperate with one teammate, constrains his actions and forces more individual solutions to emerge in the drill.

Relative to the variation of exercise duration, the $\%$ HRmax did not changed significantly. The study of Tessitore and colleagues [5] analyzed the percentage of time spent in 10-beats/minute intervals of heart rate, and it is not en-

Table 1. Mean \pm SD of $\%$ HRmax and Technical Data in all Drill Conditions

\begin{tabular}{|c|c|c|c|c|}
\hline & \multicolumn{3}{|c|}{$4 \mathrm{~min}$} & \multirow{2}{*}{$\frac{10 \mathrm{~min}}{4 \mathrm{v4}}$} \\
\hline & $4 v 4$ & $3 v 3$ & $2 \mathrm{v} 2$ & \\
\hline$\%$ HRmax & $81.5 \pm 10.6^{*}$ & $86.8 \pm 7.3$ & $87.9 \pm 11.7$ & $82.2 \pm 7.3$ \\
\hline Number of ball possessions & $2.47 \pm 0.2$ & $2.62 \pm 0.3$ & $3.31 \pm 0.5$ & $2.08 \pm 0.2$ \\
\hline Number of contacts & $2.16 \pm 0.2 * \#$ & $2.58 \pm 0.1^{*}$ & $3.85 \pm 0.4^{*}$ & $2.13 \pm 0.3 * \pi$ \\
\hline Number of dribbles & $0.47 \pm 0.13 * \#$ & $0.50 \pm 0.1 *$ & $1.31 \pm 0.1^{*}$ & $0.07 \pm 0.03 * \#$ \\
\hline Number of correct passes & $1.41 \pm 0.2$ & $2.00 \pm 0.1$ & $2.1 \pm 0.4$ & $1.25 \pm 0.1$ \\
\hline Number of wrong passes & $0.28 \pm 0.1$ & $0.29 \pm 0.1$ & $0.19 \pm 0.1$ & $0.35 \pm 0.1$ \\
\hline Number of shots on goal & $0.38 \pm 0.2$ & $0.58 \pm 0.3$ & $0.56 \pm 0.3$ & $0.36 \pm 1.0$ \\
\hline Number of tackles & $0.25 \pm 0.1^{\#}$ & $0.08 \pm 0.1$ & $0.13 \pm 0.1$ & $0.10 \pm 0.3^{\#}$ \\
\hline Number of interceptions & $0.16 \pm 0.1$ & $0.17 \pm 0.1$ & $0.41 \pm 0.1$ & $0.06 \pm 0.03$ \\
\hline
\end{tabular}

* - Statistical differences in the number of players variation; ${ }^{\text {H }}$ - Statistical differences in exercise duration variation. 


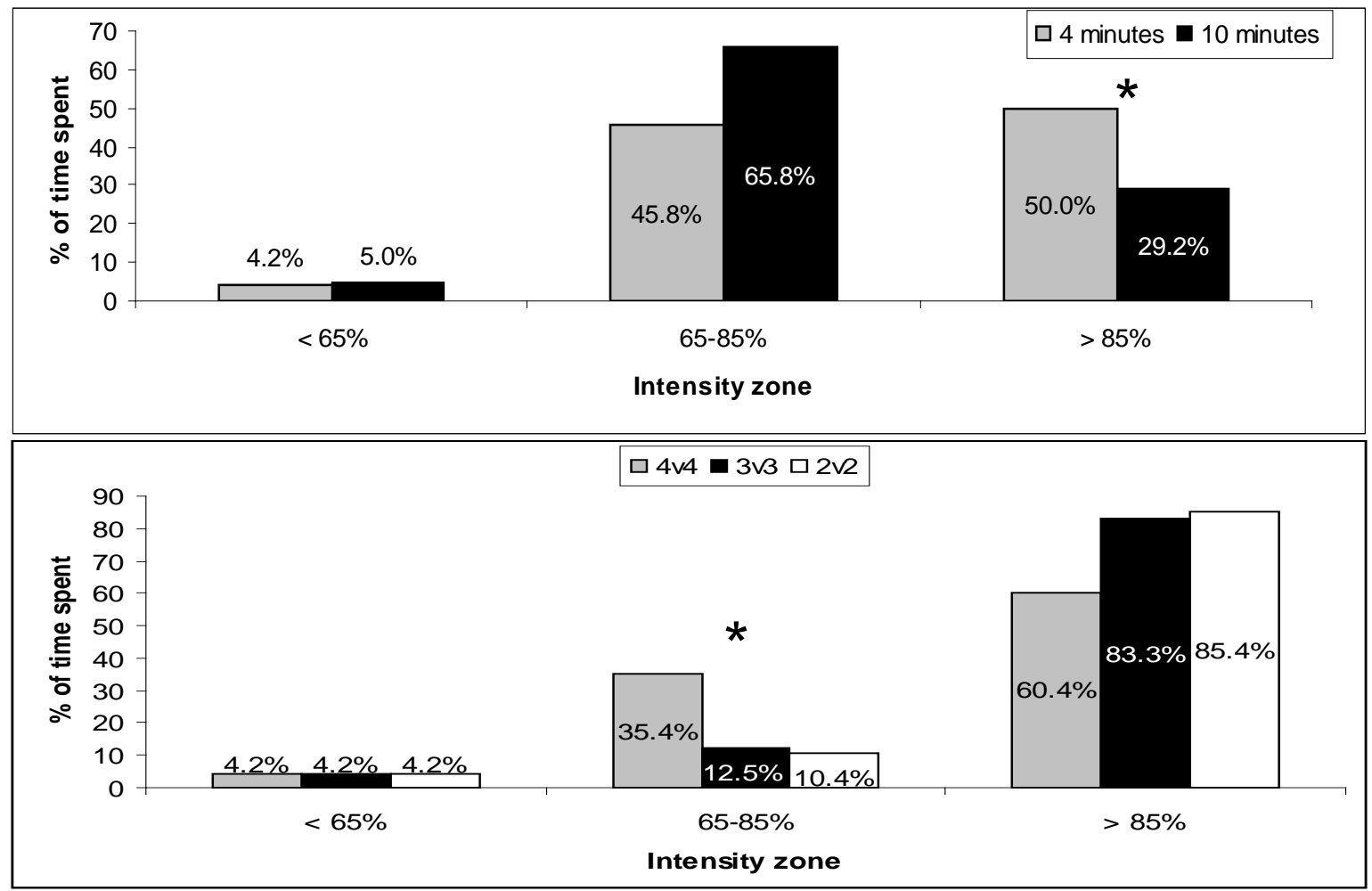

Fig. (2). Percentage of time spent in each intensity zone. Fig. 2a shows mean values relate to exercise duration. Fig. 2b shows mean values relate to players number variation. (* - Statistical differences at $\mathrm{P} \leq 0.05$ ).

lightening about the influence of the exercise duration in drill intensity. In the present study, the results showed that, although \%HRmax did not change, 4-min drill condition induced a more significant percentage of time spent in very vigorous activity (HRzone3). Thus, reducing the exercise duration, coaches can induce more elevated physiological stimulation. Some technical skills also changed by modifying exercise duration. When technical data were related to time units, the number of successive contacts with the ball, the number of dribbles, and the number of tackles were more elevated in 4-min than 10-min drill conditions. These results showed that by extending the exercise duration, the players tend to solve the game problems with less recourse to individual solutions. Concomitantly, the emergence of successive contacts with the ball, dribbles, and tackles reduce significantly.

The present study was conducted using a single squad of semi-professional players. Differences in physiological and technical response may exist between different levels of players or players with different training histories, and consequently the potential applications may not be directly transferable to other groups with different characteristics. Additionally, as limitation of the study, the reproducibility of each drill variation was not investigated.

This study was developed maintaining the ecological training settings and the practical application of our results would require that the conditions used here be adopted, including the involvement of coaches and the provision of verbal encouragement during drills. However, these findings have demonstrated that Futsal coaches can better modulate the training intensity and the emergence of some technical skills by varying the number of players and the exercise du- ration of the specific drills. It is suggested that futsal coaches can increase the physiological demands of specific drills by reducing the number of players involved and the exercise duration. These same variations can also increase the emergence of technical skills associated with solving game problems through individual solutions.

\section{ACKNOWLEDGEMENT}

The authors wish to thank at Jorge Braz for your help in the assessment procedures and methodological suggestions.

\section{REFERENCES}

[1] Barbero-Alvarez JC, Soto VM, Barbero-Alvarez V, Granda-Vera J. Match analysis and heart rate of futsal players during competition. J Sports Sci 2008; 26: 63-73.

[2] Castagna C, D’Ottavio S, Granda-Vera J, Barbero-Alvarez JC Match demands of professional Futsal: A case study. J Sci Med Sport 2008; [Epub ahead of print].

[3] Castagna C, Belardinelli R, Impellizzeri FM, Abt GA, Coutts AJ, D'Ottavio S. Cardiovascular responses during recreational 5-a-side indoor-soccer. J Sci Med Sport 2007; 10: 89-95.

[4] Rampinini E, Impellizzeri FM, Castagna C, et al. Factors influencing physiological responses to small-sided soccer games. J Sports Sci 2007; 25: 659-66.

[5] Tessitore A, Meeusen R, Piacentini MF, Demarie S, Capranica L. Physiological and technical aspects of "6-a-side" soccer drills. J Sports Med Phys Fitness 2006; 46: 36-43.

[6] Duarte R, Sampaio J, Batalha N, Maçãs V, Abrantes C. Heart rate and RPE responses to variations in Futsal specific drills. J Sports Sci 2007; 25(S2): 78-9.

[7] Folgado H, Duarte R, Laranjo L, Sampaio J, Fernandes O. Heart rate and technical responses to variation in pitch dimension and surface in "3-a-side" youth soccer drills. J Sports Sci 2007; 25(S2): 78.

[8] Gamble P. A skill-based conditioning games approach to metabolic conditioning for elite rugby football players. J Strength Cond Res 2004; 18: 491-7. 
[9] Hoff J, Wisløff U, Engen LC, Kemi OJ, Helgerud J. Soccer specific aerobic endurance training. Br J Sports Med 2002; 36: 218-21.

[10] Impellizzeri FM, Marcora SM, Castagna C, et al. Physiological and performance effects of generic versus specific aerobic interval training in soccer players. Int J Sports Med 2006; 27: 483-92.

[11] Jones S, Drust B. Physiological and technical demands of $4 \mathrm{v} 4$ and 8v8 games in elite youth soccer players. Kinesiology 2007; 39: 150-6.

[12] Little T, Williams AG. Suitability of soccer training drills for endurance training. J Strength Cond Res 2006; 20: 316-9.
[13] Gelish RL, Goslin BR, Olson RE, McDonald A, Russi GD, Moudgil VK. Longitudinal modeling of the relationship between age and maximal heart rate. Med Sci Sports Exerc 2007; 39: $822-9$.

[14] Foster C, Sykes D, Twist C, Nicholas C, Lamb K. The heart rate responses during small-sided rugby league games. J Sports Sci 2007; 25(S2): 83.

[15] Owen A, Twist C, Ford P. Small-sided games: the physiological and technical effect of altering pitch size and player numbers. Insight 2004; 7: 50-3.

(C) Duarte et al.; Licensee Bentham Open.

This is an open access article licensed under the terms of the Creative Commons Attribution Non-Commercial License (http://creativecommons.org/licenses/by$\mathrm{nc} / 3.0 /$ ) which permits unrestricted, non-commercial use, distribution and reproduction in any medium, provided the work is properly cited. 